\title{
Current materials and approaches in biomaterials and in hard tissue repair
}

Biomaterials field have been revealing significant developments in various directions. One such area is hard tissue repair and regeneration which has been the focus of intensive research in recent years especially because of the new materials that were made available in the last 10-15 years. Major advances were observed in biodegradable materials, their use in the hard tissue implants area gained impetus and also a new field, Tissue Engineering, emerged. The best known compound among the synthetic biodegradable materials is the polyester polylactide (PLA), and its various isomers and copolymers with glycolic acid (PLGA). Recently, polyhydroxyalkanoates, PHA, the natural biodegradable polyesters that are produced mainly through microbial route and also by genetically altered plants, started drawing attention in hard tissue repair studies. PHA production is through biotechnological methods and is therefore advantageous because they are more environment friendly than comparable polyesters. The biodegradable hard tissue implants of either synthetic or biological origin need not be removed after tissue repair. They do not produce the adverse effects, such as bone resorption or stress concentration, caused by metallic or ceramic implants due to the incompatibility of their physical properties (density, hardness, etc) with those of the body. They, however, lack adequate mechanical properties essential for use in cortical bone applications. Incorporation of various materials like hydroxyapatite which have high compressive strength and hardness or use of various processing approaches to improve the properties of these implants is another field of extensive research. Based on all these, last year it was decided to have hard tissue implants and repair as the main theme of the 8th Biomedical Science and Technology Symposium (Biomed 8) that was held in Ankara during 5-8 September 2001.

Among the other major topics during the Symposium Tissue Engineering had a special place. This topic is making a great impact in the biomaterials area. It involves preparation of a porous structure preferably of biodegradable materials and their loading with cells of the type of the target tissue into which implantation will be carried out. For example, when bone is the target site then the cells in question are generally osteoblasts. The porous structure or the "matrix" is very important because in order for the implanted cells to adhere, proliferate, and differentiate and thus create a tissue one needs a surface with appropriate architecture, porosity, cell bonding capability and biodegradability. For this purpose the above mentioned biodegradable polymers are used after surface modifications (chemical, or with rf-plasma), by blending with other materials or after attachment of side groups like RGD sequences, PEG, heparin, etc. enabling them to mimic the extracellular matrix. The developments in the area have taught us that cell-implant interaction is the most important parameter that determines the success of the implant.

There were various other important topics covered at the Symposium. For example cellular products, like bone or epithelial growth factors, bone morphogenic protein, etc. contribute significantly to the healing of bone and other tissues. Their controlled release led to new applications of controlled bioactive 
agent delivery. These controlled release systems are especially conveniently applied when the implants used along with these factors are polymeric or carry a polymer coat that could carry the agent in it. Another major area to which controlled drug delivery approaches contributed significantly is gene therapy. Delivery of these labile genetic macromolecules need great care and appropriate designs to be succesful and thus were a new application of controlled drug delivery.

Fixation of hard tissue implants still retained the interest it has generated years ago. This is mainly because cemented fixation still had its inherent problems of exothermicity and resultant tissue necrosis.

General biomaterial development and characterization is always exciting both due to the new techniques made available to the biomaterials scientist and because new compounds or blends are continuously developed. These were also presented at the Symposium.

In conclusion, it was encouraging to see that the biomaterials field remains a very exciting area to be involved in and this Symposium renewed our confidence in this belief. I am grateful to all the participants who came from near (Turkey) and far (Finland, USA, Portugal, UK) for keeping the interest in this very exciting field alive by their valuable contributions. I also wish to express my sincere thanks to Prof A.E. Engin and Technology and Health Care journal for enabling our scientific contributions to reach a much larger audience than it normally would.

Vasif Hasircl

Middle East Technical University

Ankara, Turkey 